\section{Bär und Leu}

Eine Zirkusnummer? - Mitnichten!

Eine Bankenfusion? - Nicht unsere Sorge!

Bär und Leu - ein Kreis von Ärzten im Raum Bern und Zentralschweiz (Bär), mit dem Präsidenten Dr. med. H. R. Brunner, Chefarzt für Anästhesie am Kantonsspital Baden, und eine Gruppe von freiwilligen Frauen, die "Marienvereinigung", unter der Leitung der Ärztin Frau Dr. Nadja Khoma in Lemberg (Leu).

Gemeinsam möchten sie einen Mittagstisch für bedürftige Kinder einrichten. Die wirtschaftliche Lage in der Ukraine ist zehn Jahre nach dem Ende der Sowjetherrschaft desolat. Das soziale Netz, das zu Zeiten des Kommunismus noch einigermassen funktionierte, ist zerrissen:
- in Lemberg (800000 Einwohner) sind schätzungsweise 4000-5000 Kinder ohne elterliche Pflege und Aufsicht.

- 50\% aller Kinder weisen Entwicklungsstörungen auf, die unter anderem auf die Mangelernährung zurückzuführen sind.

- $\quad$ 40\% aller Familien haben ein Auskommen, das unterhalb der Armutsgrenze liegt.

Fühlen Sie sich angesprochen? Möchten Sie helfen, den Hunger eines notleidenden Kindes zu stillen? Für Beiträge in jeglicher Höhe steht Ihnen unser Konto bei der Raiffeisenbank Thunersee-Süd zur Verfügung, Konto-Nr. 7916846-80838. Postcheck 30-2028-5.

Gerne beantwortet der Sekretär von «Bär und Leu» Ihre Fragen: Dr. H. Vogt, "Brüggetli», 3704 Krattigen, Tel./Fax 0336542281.

\title{
www.engelskinder.ch
}

\section{K. Favero}

Es gibt viele Frauen, bei denen die Schwangerschaft ohne guten Ausgang endet. Es gibt viel mehr als man meint. Auch heute ist es immer noch ein Tabuthema, wenn man ein Kind verliert. Dieses Tabu sollte gebrochen werden, daher die Idee zu dieser Homepage.

Ich selber habe am 23. März 1999 meinen Sohn in der 21. Schwangerschaftswoche tot geboren. Ich bekam keine Hilfe, keine Bücherlisten, einfach nichts.

Korrespondenz:

Katharina Favero

Brandrietstrasse 1

CH-8307 Effretikon
Hätte ich damals schon gewusst, dass es im Internet eine Seite (aus Deutschland) für betroffene Eltern gibt, wäre mir sehr geholfen gewesen. Ich war alleine und kannte niemanden mit dem gleichen Schicksal; ich wusste aber, dass es viele gibt. Am 12. April 2000 habe ich eine Homepage für Eltern, die ihre Kinder durch Fehl-, Früh- oder Totgeburt verloren haben, unter www.engelskinder.ch aufgeschaltet. Die Homepage wird täglich von vielen neuen Betroffenen besucht (jetzt schon über 3200 Besucherinnen und Besucher), und es zeigt mir, dass es notwendig war, eine solche Site anzubieten. Man weiss, dass man nicht alleine und nicht die einzige ist mit dieser Erfahrung. Das hilft einfach schon viel. Ich bekomme täglich $\mathrm{Zu}-$ schriften von Betroffenen, die mir dafür danken, und daher finde ich es wichtig, dass auch die Ärzte von dieser Homepage Kenntnis haben, damit sie die Adresse ihren betroffenen Patientinnen weitergeben können. 